\title{
WEIERSTRASS SEMIGROUPS ON DOUBLE COVERS OF PLANE CURVES OF DEGREE 5
}

\author{
SEON JeONG Kim and JiRyo Komeda
}

\begin{abstract}
We investigate Weierstrass semigroups of ramification points on double covers of plane curves of degree $d$. Using the results we determine all the Weierstrass semigroups in the case $d=5$ when the genus of the covering curve is greater than 17 and the ramification point is on a non-ordinary flex.
\end{abstract}

\section{Introduction}

Let $C$ be a smooth irreducible curve of genus $g$, where a curve means a projective curve over an algebraically closed field of characteristic 0 . For a point $P$ of $C$ we define the Weierstrass semigroup $H(P)$ of $P$ as follows:

$H(P)=\left\{n \in \mathbf{N}_{0} \mid\right.$ there is a rational function $f$ on $C$ such that $\left.(f)_{\infty}=n P\right\}$,

where $\mathbf{N}_{0}$ is the additive monoid of non-negative integers and $(f)_{\infty}$ means the polar divisor of $f$. Then $H(P)$ is a numerical semigroup of genus $g$, which means a submonoid of $\mathbf{N}_{0}$ whose complement is a finite set with cardinality $g$. The genus of a numerical semigroup $H$ is denoted by $g(H)$. For a numerical semigroup $H$ we denote by $d_{2}(H)$ the set consisting of the elements $h$ for $2 h \in H$, which is a numerical semigroup. For positive integers $a_{1}, \ldots, a_{s}$ we denote by $\left\langle a_{1}, \ldots, a_{s}\right\rangle$ the additive monoid generated by $a_{1}, \ldots, a_{s}$.

We will study about the numerical semigroups $H$ which are the Weierstrass semigroups of ramification points on double covers of smooth plane curves of degree $d$. In this paper such a numerical semigroup $H$ is said to be of double covering type of a plane curve, which is abbreviated to $D C P$. In this case, $d_{2}(H)$ is the Weierstrass semigroup of a point on a smooth plane curve of degree $d$. If $d_{2}(H)$ is the Weierstrass semigroup of a point on a smooth plane curve of degree $d \leqq 3$, i.e., $d_{2}(H)=\mathbf{N}_{0}$ or $\langle 2,3\rangle$, then we can show that $H$ is DCP (for

2010 Mathematics Subject Classification. 14H55, 14H30, 20 M14.

Key words and phrases. Numerical semigroup, Weierstrass semigroup of a point, Double cover of a plane curve, Plane curve of degree 5.

Received June 4, 2014; revised September 26, 2014. 
example, see [8]). In the case $d=4$, i.e., $d_{2}(H)=\langle 3,4\rangle,\langle 3,5,7\rangle$ or $\langle 4,5,6,7\rangle$ the papers [9], [4], [5] and [6] show that every numerical semigroup $H$ with $g(H) \geqq 6$ is DCP except $H=\langle 8,10,12,14, n, n+4\rangle$ with odd $n \geqq 9, \quad H=$ $\langle 7 \rightarrow 10,12\rangle$ and $H=\langle 5,7,8\rangle$. The excluded semigroups are not DCP.

Let $C$ be a smooth plane curve and $P$ its point. Let $Z$ be a plane curve. We denote by $C . Z$ the intersection divisor of $C$ with $Z$. Moreover, let $\operatorname{ord}_{P}(C . Z)$ be the multiplicity of $C . Z$ at $P$. We denote by $T_{P}$ the tangent line at $P$ on $C$. We note the following:

i) If $P$ is a total flex on a smooth plane curve $C$ of degree 5, i.e., $\operatorname{ord}_{P} C . T_{P}=5$, then $H(P)=\langle 4,5\rangle$.

ii) If $P$ is a point with $\operatorname{ord}_{P} C \cdot T_{P}=4$ on a smooth plane curve $C$ of degree 5, then $H(P)=\langle 4,7,10,13\rangle$.

The following is the main result of this article:

MAIN THEOREM. Let $H$ be a numerical semigroup of genus $\geqq 18$.

i) If $d_{2}(H)=\langle 4,5\rangle$, then $H$ is $D C P$.

ii) Assume that $d_{2}(H)=\langle 4,7,10,13\rangle$. If $H$ is distinct from $2 d_{2}(H)+$ $\langle n, n+4\rangle$ and $2 d_{2}(H)+\langle n, n+12\rangle$ with odd $n \geqq 13$, then it is DCP. The excluded semigroups are not DCP.

Corollary 2.7 in Section 2 shows i) in the above theorem. Corollary 3.2 in Section 3 and Theorem 4.2 in Section 4 mean ii) in Main Theorem.

\section{Ramification points over total flexes}

A numerical semigroup $H$ is called an a-semigroup if the least positive integer in $H$ is $a$. For an $a$-semigroup $H$ we set $S(H)=\left\{a, s_{1}, \ldots, s_{a-1}\right\}$ where $s_{i}=\min \{h \in H \mid h \equiv i \bmod a\}$, which is called the standard basis for $H$. Let $d$ be an integer which is larger than 2 . In this section we set

$$
H_{d}=\langle d-1, d\rangle \text { and } s_{i}=i d \text { for } 1 \leqq i \leqq d-2 .
$$

Then we have $S\left(H_{d}\right)=\left\{d-1, s_{1}, s_{2}, \ldots, s_{d-2}\right\}$.

First we will show that eight kinds of numerical semigroups $H$ with $d_{2}(H)=\langle d-1, d\rangle$ are DCP. We use the following lemma when we calculate the genera $g(H)$ of such numerical semigroups $H$.

LeMmA 2.1. Let $m$ and $l$ be positive integers with $2 \leqq m \leqq d-1$ and $l \leqq((d-m) d) /(d-1)$. Let $n$ be an odd number with $n \geqq d(\bar{d}-2)$. Set

$$
H=2 H_{d}+\left\langle n, n+2 s_{d-m}-2 l(d-1)\right\rangle .
$$

Then

$$
H=\left(2 H_{d}+n \mathbf{N}_{0}\right) \cup\left\{n+s_{d-i}-2 j(d-1) \mid 2 \leqq i \leqq m, 1 \leqq j \leqq l\right\},
$$

which implies that $g(H)=(d-1)(d-2)+(n-1) / 2-l(m-1)$. 
Proof. By the assumption on $n$ and Remark 2.1 in [7] we have

$$
S\left(2 H_{d}+n \mathbf{N}_{0}\right)=\left\{2(d-1), 2 s_{1}, \ldots, 2 s_{d-2}, n, n+2 s_{1}, \ldots, n+2 s_{d-2}\right\} .
$$

Assume that $n+2 s_{d-m-1}-2(d-1)$ belongs to $H$. Then the element $s \in S(H)$ with $s \equiv n+2 d(d-m-1) \bmod 2(d-1)$ is written by

$$
s=n+2 s_{d-m}-2 l(d-1)+t,
$$

where $t$ is the minimum in $H=2 H_{d}+\left\langle n, n+2 s_{d-m}-2 l(d-1)\right\rangle$ with $t \equiv$ $2 d(d-2) \bmod 2(d-1)$. Since

$$
2\left(n+2 s_{d-m}-2 l(d-1)\right)-2 s_{d-2}=2\left(n-s_{d-2}\right)+4\left(s_{d-m}-l(d-1)\right) \geqq 0
$$

by the assumptions $n \geqq d(d-2)$ and $l \leqq((d-m) d) /(d-1)$, we have $t=2 s_{d-2}$. Hence, we get $n+2 s_{d-m-1}-2(d-1) \geqq n+2 s_{d-m}-2 l(d-1)+2 s_{d-2}$, which implies that $(l-1)(d-1) \geqq(d-1) d$. Thus, we have $l \geqq d+1$. Then the assumption on $l$ induces $d+1 \leqq l \leqq((d-m) d) /(d-1)$, which implies that

$$
d^{2}-1<(d-m) d \leqq(d-2) d=d^{2}-2 d .
$$

This is a contradiction. Therefore, we obtain $n+2 s_{d-m-1}-2(d-1) \notin H$.

Moreover, we will show that $n+2 s_{d-2}-2(l+1)(d-1) \notin H$. Assume that $n+2 s_{d-2}-2(l+1)(d-1) \in H$. Then the element $s \in S(H)$ with $s \equiv$ $n+2 d(d-2) \bmod 2(d-1)$ is written by

$$
s=n+2 s_{d-m}-2 l(d-1)+t,
$$

where $t$ is the minimum in $H=2 H_{d}+\left\langle n, n+2 s_{d-m}-2 l(d-1)\right\rangle$ with $t \equiv$ $2 d(m-2) \bmod 2(d-1)$. Since

$$
\begin{aligned}
2(n+ & \left.2 s_{d-m}-2 l(d-1)\right)-2 s_{m-2} \\
& =2 n-4 l(d-1)+2 d(2 d-3 m+2) \geqq 2 d(d-m) \geqq 2 d>0
\end{aligned}
$$

by the assumptions $n \geqq d(d-2), l \leqq((d-m) d) /(d-1)$ and $m \leqq d-1$, we have $t=2 s_{m-2}$. Hence, we get

$$
n+2 s_{d-2}-2(l+1)(d-1) \geqq n+2 s_{d-m}-2 l(d-1)+2 s_{m-2},
$$

which implies that $1 \geqq d$. This is a contradiction.
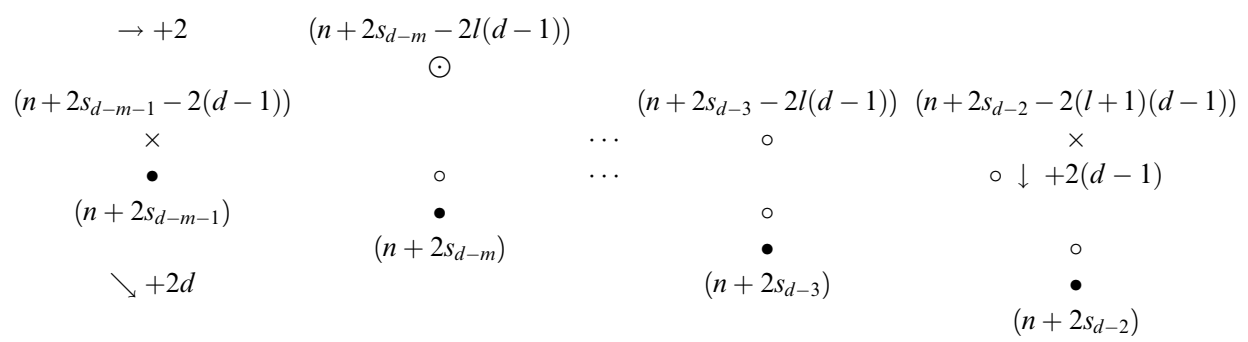

The elements of $H=2 H_{d}+\left\langle n, n+2 s_{d-m}-2 l(d-1)\right\rangle$ 
Let $i \geqq 2$ and $j \geqq l+1$. Then

$$
\begin{gathered}
n+2 s_{d-2}-2(l+1)(d-1)-\left(n+2 s_{d-i}-2 j(d-1)\right) \\
=2(i-2) d+2(j-l-1)(d-1) \in 2 H_{d} .
\end{gathered}
$$

Since $n+2 s_{d-2}-2(l+1)(d-1) \notin H$, we must have $n+2 s_{d-i}-2 j(d-1) \notin H$. Let $i \geqq m+1$ and $j \geqq 1$. Then

$$
\begin{gathered}
n+2 s_{d-m-1}-2(d-1)-\left(n+2 s_{d-i}-2 j(d-1)\right) \\
=2(i-m-1) d+2(j-1)(d-1) \in 2 H_{d} .
\end{gathered}
$$

Since $n+2 s_{d-m-1}-2(d-1) \notin H$, we obtain $n+2 s_{d-i}-2 j(d-1) \notin H$. Hence, the largest odd number $n^{\prime}$ in the complement of $H$ is $n+2 s_{d-m-1}-2(d-1)$ or $n+2 s_{d-2}-2(l+1)(d-1)$ and $g\left(H+\left\langle n^{\prime}\right\rangle\right)=g(H)-1$, which follows from the above figure. Thus, we have

$$
H=\left(2 H_{d}+n \mathbf{N}_{0}\right) \cup\left\{n+2 s_{d-i}-2 j(d-1) \mid 2 \leqq i \leqq m, 1 \leqq j \leqq l\right\},
$$

because $H \backslash 2 H_{d}$ contains no even number. Since we have $g\left(2 H_{d}+n \mathbf{N}_{0}\right)=$ $(d-1)(d-2)+(n-1) / 2$, we get our desired result.

In the rest of this section we are in the following situation: Let $C$ be a smooth plane curve of degree $d \geqq 5$ with a point $P$ satisfying $\operatorname{ord}_{P} C \cdot T_{P}=d$, i.e., $H(P)=H_{d}$. We state the following Namba's famous lemma (see Lemma 2.3.2 in [12]), since it plays an important role in the calculation of $\operatorname{ord}_{P}\left(C_{1} . C_{2}\right)$, which is the intersection multiplicity of plane curves $C_{1}$ and $C_{2}$ at $P$.

Lemma 2.2. Let $C_{1}, C_{2}$ and $C_{3}$ be plane curves. Assume that $C_{1}$ is irreducible and is neither a component of $C_{2}$ nor of $C_{3}$. Let $P$ be a smooth point of $C_{1}$. Then

$$
\operatorname{ord}_{P}\left(C_{2} \cdot C_{3}\right) \geqq \min \left\{\operatorname{ord}_{P}\left(C_{1} \cdot C_{2}\right), \operatorname{ord}_{P}\left(C_{1} \cdot C_{3}\right)\right\} .
$$

The following lemma is useful for determining the Weierstrass semigroup of a ramification point on a double cover of a plane curve.

Lemma 2.3. Let $C_{d-3}$ be a plane curve of degree $d-3$ such that $\operatorname{ord}_{P}\left(C_{d-3} \cdot C\right) \geqq(d-3-l) d$ with an integer $l \leqq d-4$. Then $C_{d-3}=T_{P}^{d-3-l} C_{l}$, where $C_{l}$ is a plane curve of degree $l$, which implies that $\operatorname{ord}_{P}\left(C_{l} . C\right) \geqq$ $\operatorname{ord}_{P}\left(C_{d-3} \cdot C\right)-(d-3-l) d$.

Proof. We have $\operatorname{ord}_{P}\left(T_{P}^{d-3-l} \cdot C\right)=(d-3-l) d$. Hence, by the assumption and Lemma 2.2 we get $\operatorname{ord}_{P}\left(C_{d-3} \cdot T_{P}^{d-3-l}\right) \geqq(d-3-l) d$. Thus, we have $C_{d-3}=T_{P} C_{d-4}$, where $C_{d-4}$ is a plane curve of degree $d-4$. Moreover, we get

$$
\operatorname{ord}_{P}\left(C_{d-4} \cdot C\right) \geqq(d-4-l) d \quad \text { and } \quad \operatorname{ord}_{P}\left(T_{P}^{d-4-l} \cdot C\right)=(d-4-l) d,
$$

which implies that $\operatorname{ord}_{P}\left(C_{d-4} \cdot T_{P}^{d-4-l}\right) \geqq(d-4-l) d$. Hence, we get $C_{d-3}=$ $T_{P}^{2} C_{d-5}$ if $d-4-l \geqq 1$, where $C_{d-5}$ is a plane curve of degree $d-5$. Using this 
method successively we get $C_{d-3}=T_{P}^{d-3-l} C_{l}$, where $C_{l}$ is a plane curve of degree $l$.

To prove that a numerical semigroup is DCP we use the following theorem many times, which is stated in Theorem 2.2 of [9].

THEOREM 2.4. Let $H$ be a numerical semigroup. Set

$$
n=\min \{h \in H \mid h \text { is odd }\} \text {. }
$$

Then

$$
g(H)=2 g\left(d_{2}(H)\right)+(n-1) / 2-r
$$

with some non-negative integer $r$ (for example, see Lemma 3.1 in [4]). Assume that $d_{2}(H)$ is Weierstrass. Take a pointed curve $(C, P)$ with $H(P)=d_{2}(H)$. Let $Q_{1}, \ldots, Q_{r}$ be points of $C$ different from $P$ with $h^{0}\left(Q_{1}+\cdots+Q_{r}\right)=1$. Moreover, assume that $H$ has an expression

$$
H=2 d_{2}(H)+\left\langle n, n+2 l_{1}, \ldots, n+2 l_{s}\right\rangle
$$

of generators with positive integers $l_{1}, \ldots, l_{s}$ such that

$$
h^{0}\left(l_{i} P+Q_{1}+\cdots+Q_{r}\right)=h^{0}\left(\left(l_{i}-1\right) P+Q_{1}+\cdots+Q_{r}\right)+1
$$

for all $i$. If the divisor $n P-2 Q_{1}-\cdots-2 Q_{r}$ is linearly equivalent to some reduced divisor not containing $P$, then there is a double covering $\pi: \tilde{C} \rightarrow C$ with a ramification point $\tilde{P}$ over $P$ satisfying $H(\tilde{P})=H$.

We may replace the assumption in Theorem 2.2 of [9] that the complete linear system $\left|n P-2 Q_{1}-\cdots-2 Q_{r}\right|$ is base point free by the above assumption that the divisor $n P-2 Q_{1}-\cdots-2 Q_{r}$ is linearly equivalent to some reduced divisor not containing $P$, because the same proof as in Theorem 2.2 of [9] works well under our assumption.

THEOREM 2.5. Let $n$ be an odd number with $n \geqq d(d-2)$. Let $H_{d}$ denote $H(P)=\langle d-1, d\rangle$. Let $H$ be a numerical semigroup which is one of the following:

(i) $2 H_{d}+\left\langle n, n+2 t_{1}\right\rangle$ with $t_{1}=s_{d-2}-l(d-1)$ where $l$ is a positive integer with $l \leqq d-2$ and $n \geqq(d-1)(d-2)+1+2 l$.

(ii) $2 H_{d}+\left\langle n, n+2 t_{1}\right\rangle$ with $t_{1}=s_{d-m}-(d-1)$ where $m$ is an integer with $2 \leqq m \leqq d-1$ and $n \geqq(d-1)(d-2)-1+2 m$.

(iii) $2 H_{d}+\left\langle n, n+2 t_{1}\right\rangle$ with $t_{1}=s_{d-m}-2(d-1)$ where $m$ is an integer with $2 \leqq m \leqq d-2$ and $n \geqq(d-1)(d-2)-3+4 m$.

(iv) $2 H_{d}+\left\langle n, n+2 t_{1}, n+2 t_{2}\right\rangle$ with $t_{1}=s_{d-2}-2(d-1)$ and $t_{2}=s_{d-m}-$ $(d-1)$ where $m$ is an integer with $3 \leqq m \leqq d-1$ and $n \geqq(d-1)(d-2)$ $+1+2 m$.

(v) $2 H_{d}+\left\langle n, n+2 t_{1}, n+2 t_{2}\right\rangle$ with $t_{1}=s_{d-2}-l(d-1)$ and $t_{2}=s_{d-3}-$ $(d-1)$ where $l$ is an integer with $3 \leqq l \leqq d-2$ and $n \geqq(d-1)(d-2)$ $+3+2 l$. 
(vi) $2 H_{d}+\left\langle n, n+2 t_{1}, n+2 t_{2}\right\rangle$ with $t_{1}=s_{d-4}-(d-1)$ and $t_{2}=s_{d-2}-$ $3(d-1)$ where $n \geqq(d-1)(d-2)+11$.

(vii) $2 H_{d}+\left\langle n, n+2 t_{1}, n+2 t_{2}\right\rangle$ with $t_{1}=s_{d-3}-2(d-1)$ and $t_{2}=s_{d-2}-$ $3(d-1)$ where $n \geqq(d-1)(d-2)+11$.

(viii) $2 H_{d}+\left\langle n, n+2 t_{1}, n+2 t_{2}\right\rangle$ with $t_{1}=s_{d-4}-(d-1)$ and $t_{2}=s_{d-3}-$ $2(d-1)$ where $n \geqq(d-1)(d-2)+11$.

Then $H$ is $D C P$.

Proof. To prove that $H$ is DCP we use Theorem 2.2 in [9]. We show step by step that $H$ satisfies the conditions of the theorem in [9].

SteP 1. By Lemma 2.1 we have

$$
g(H)=(d-1)(d-2)+\frac{n-1}{2}-r
$$

where (i) $r=l$, (ii) $r=m-1$ and (iii) $r=2(m-1)$.

(iv) We note that $n+2 s_{d-2}-4(d-1)$ is the largest number in the complement of the semigroup $2\langle d-1, d\rangle+\left\langle n, n+2 s_{d-m}-2(d-1)\right\rangle$ in $\mathbf{N}_{0}$ (see the figure below).
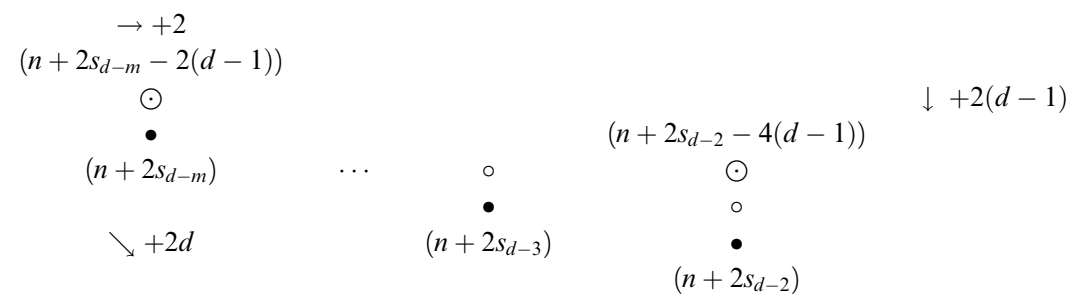

The elements of $H=2\langle d-1, d\rangle+\left\langle n, n+2 s_{d-2}-4(d-1), n+2 s_{d-m}-2(d-1)\right\rangle$

Using Lemma 2.1 we get $r=m$.

(v) We have $r=l+1$. In fact, we note that $n+2 s_{d-3}-2(d-1)-\left(n+2 s_{d-2}-2(l+1)(d-1)\right)=2((l-1)(d-1)-1)>0$, which implies that $n+2 s_{d-3}-2(d-1)$ is the largest number in the complement of the semigroup $2\langle d-1, d\rangle+\left\langle n, n+2 s_{d-2}-2 l(d-1)\right\rangle$ in $\mathbf{N}_{0}$. Using Lemma 2.1 we get $r=l+1$.

(vi) We have $r=5$. In fact, we have the following figure:

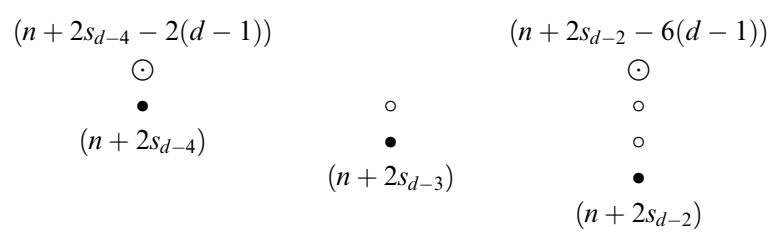


(vii) We have $r=5$. In fact, we have the following figure:

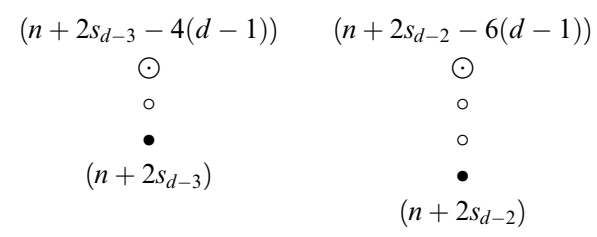

(viii) We have $r=5$. In fact, we have the following figure:

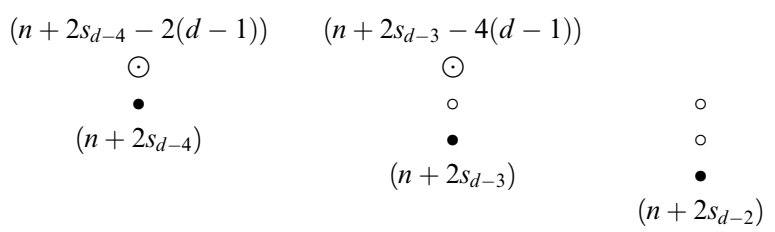

SteP 2. Let $E$ be a divisor of degree $n-2 r$ on a smooth plane curve of degree $d$. By the assumption on $n$ in each case we have $\operatorname{deg} E \geqq$ $(d-2)(d-1)+1$, which implies that $E$ is very ample.

STEP 3. In each case we choose $r$ points $Q_{1}, \ldots, Q_{r}$ of $C$ with $Q_{i} \neq P$ for all $i$ satisfying the equality

$$
h^{0}\left(Q_{1}+\cdots+Q_{r}\right)=1 .
$$

To choose the points $Q_{1}, \ldots, Q_{r}$ satisfying the equality (1) we use the following: Let $P_{1}, \ldots, P_{k}(k \leqq d)$ be points on a smooth plane curve of degree $d \geqq 4$. Then $h^{0}\left(P_{1}+\cdots+P_{k}\right)=1$ unless $k \geqq d-1$ and at least $d-1$ points of $P_{1}, \ldots, P_{k}$ are collinear. This follows from the fact that a smooth plane curve of degree $d \geqq 4$ is $(d-1)$-gonal and has a unique $g_{d}^{2}$, which is cut out by lines. The latter fact is called Namba's Theorem (see [12]).

(i) Let us take a line $L$ with $L \not \nexists P$. We set $L . C=Q_{1}+\cdots+Q_{d}$ with $Q_{i} \neq P$ for all $i$. Since $C$ is $(d-1)$-gonal (see [1]), we have the equality (1) because $r=l \leqq d-2$.

(ii) Let $L$ be a line through $P$ distinct from the tangent line $T_{P}$. We set $L . C=P+Q_{1}+\cdots+Q_{d-1}$ with $Q_{i} \neq P$, all $i$. Then we have the equality (1) because $r=m-1 \leqq d-2$.

(iii) Take two distinct lines $L_{1}$ and $L_{2}$ through $P$ different from $T_{P}$. We set

$$
L_{1} \cdot C=P+R_{1}+\cdots+R_{d-1} \quad \text { and } \quad L_{2} \cdot C=P+S_{1}+\cdots+S_{d-1} .
$$

Then

$$
h^{0}\left(R_{1}+\cdots+R_{m-1}+S_{1}+\cdots+S_{m-1}\right)=1 .
$$

Indeed, we have

$$
\left|R_{1}+\cdots+R_{m-1}+S_{1}+\cdots+S_{m-1}\right|=\left|L_{1}+L_{2}-E\right|,
$$


where $E$ is an effective divisor of degree $\geqq 6$. It is known that a complete linear system of degree at most $2 d-5$ on a smooth plane curve of degree $d \geqq 4$ is zero-dimensional or empty unless its free part is a $g_{d-1}^{1}$ or a $g_{d}^{2}$ (and hence it contains at least $d-1$ collinear points). This fact follows from Theorem 3.1 in [3]. Hence we get the equality (2). We set $Q_{i}=R_{i}$ for $i=1, \ldots, m-1$ and $Q_{m-1+i}=S_{i}$ for $i=1, \ldots, m-1$. Hence we get the equality (1).

(iv) Let $L$ be a line through $P$, distinct from $T_{P}$. We set L.C= $P+R_{1}+\cdots+R_{d-1}$. For $i=1, \ldots, m-1$ we set $Q_{i}=R_{i}$ and take a point $Q_{m} \in C$ which is not in $L$. If $r=m \leqq d-2$, we get the equality (1). Hence, we may assume that $m=d-1$. Since $Q_{1}, \ldots, Q_{d-1}$ are not collinear, we get the equality (1).

(v) Let $L$ be a line not passing through $P$ with $L . C=R_{1}+\cdots+R_{d}$. We set $Q_{i}=R_{i}$ for $i=1, \ldots, l$. Choose $Q_{l+1} \in C$ with $Q_{l+1} \neq P$ and $Q_{l+1} \notin L$. By the same way as in (iv) we get the equality (1).

(vi) Let $L$ be a line through $P$ with $L \neq T_{P}$ and $L . C=P+R_{1}+\cdots+R_{d-1}$. We set $Q_{1}=R_{1}, Q_{2}=R_{2}$ and $Q_{3}=R_{3}$. Take two distinct points $Q_{4}$ and $Q_{5}$ of $C$ which do not belong to the line $L$ such that the line $L_{Q_{4}, Q_{5}}$ through $Q_{4}$ and $Q_{5}$ does not contain $P$. It suffices to show the equality in the case $d=5,6$. Let $d=5$. Take a curve $C_{2}$ of degree 2 with $C_{2} . C \geqq Q_{1}+\cdots+Q_{5}$. Since we have $L . C \geqq Q_{1}+Q_{2}+Q_{3}$, we get $C_{2} . L \geqq Q_{1}+Q_{2}+Q_{3}$. Hence, we have $C_{2}=L L_{1}$ where $L_{1}$ is a line. Since the line $L$ contains neither $Q_{4}$ nor $Q_{5}$, a line $L_{1}$ must contain $Q_{4}$ and $Q_{5}$. Hence, $L_{1}$ is uniquely determined. Thus, $C_{2}$ is uniquely determined. Therefore, we get $h^{0}\left(Q_{1}+\cdots+Q_{5}\right)=1$. Let $d=6$. The points $Q_{1}, \ldots, Q_{5}$ are not collinear. Hence we obtain the equality (1).

(vii) Let $Q_{1}, \ldots, Q_{5}$ be general points. Then we get the equality (1).

(viii) Let $L_{1}$ be a line through $P$ with $L_{1} \neq T_{P}$ and $L_{1} . C=P+R_{1}+\cdots+$ $R_{d-1}$. We set $Q_{1}=R_{1}$ and $Q_{2}=R_{2}$. Take a point $Q_{3}$ of $C$ which does not belong to the line $L_{1}$. Let $L_{2}$ be the line through $Q_{3}$ and $P$. We set $L_{2}$. $C=$ $P+Q_{3}+S_{1}+\cdots+S_{d-2}$. Let $Q_{4}=S_{1}$ and $Q_{5}=S_{2}$. Then we have $Q_{4}, Q_{5} \notin$ $\left\{Q_{1}, Q_{2}\right\}$ and $L_{2} . C ¥ Q_{i}$ for $i=1,2$. It suffices to show the equality in the case $d=5,6$. Let $d=5$. Let $C_{2}$ be a conic with $C_{2} . C \geqq Q_{1}+\cdots+Q_{5}$. Since $L_{2} . C \geqq Q_{3}+Q_{4}+Q_{5}$, we obtain $C_{2}=L_{2} L$ where $L$ is a line. Now we have $Q_{1}+\cdots+Q_{5} \leqq L_{2} . C+L . C$, which implies that $L . C \geqq Q_{1}+Q_{2}$. Hence, we get $L=L_{1}$. Thus, a conic $C_{2}$ is uniquely determined. Let $d=6$. The points $Q_{1} \ldots, Q_{5}$ are not collinear. Thus, we have the equality (1).

STEP 4. We set $D_{r}=Q_{1}+\cdots+Q_{r}$. In this step $C_{i}$ means a plane curve of degree $i$. We will show that

$$
h^{0}\left(K-t_{i} P-D_{r}\right)=h^{0}\left(K-\left(t_{i}-1\right) P-D_{r}\right)
$$

for $i=1,2$ where $K$ is a canonical divisor on $C$. Let $C_{d-3} . C \geqq\left(t_{i}-1\right) P+D_{r}$. It suffices to show that $C_{d-3} . C \geqq t_{i} P+D_{r}$ because of the fact that $H^{0}\left(\mathbf{P}^{2}, \mathcal{O}_{\mathbf{P}^{2}}(d-3)\right) \simeq H^{0}\left(C, \mathcal{O}_{C}(K)\right)$. 
(i) By Lemma 2.3 we obtain $C_{d-3}=T_{P}^{d-2-l} C_{l-1}$. Hence, we have $T_{P}^{d-2-l} \cdot C+C_{l-1} \cdot C \geqq\left(t_{1}-1\right) P+D_{r}=(d-2-l) d P+(l-1) P+D_{r}$,

which implies that $C_{l-1} . C \geqq(l-1) P+D_{r}$. Thus, we get $L . C_{l-1} \geqq D_{r}$ where $L$ is as in Step 3. In view of $r=l$ we get $C_{l-1}=L C_{l-2}$, which implies that $C_{d-3}=$ $T_{P}^{d-2-l} L C_{l-2}$. Moreover, we obtain

$$
(d-2-l) d P+Q_{1}+\cdots+Q_{d}+C_{l-2} \cdot C \geqq((d-2-l) d+l-1) P+D_{r} .
$$

Hence, we get $C_{l-2} . C \geqq(l-1) P$. Since $T_{P} . C=d P$ and $d-3 \geqq l-1$, we have $C_{l-2} . T_{P} \geqq(l-1) P$, which implies that $C_{l-2}=T_{P} C_{l-3}$. Thus, we get $C_{d-3}=$ $T_{P}^{d-1-l} L C_{l-3}$. Therefore, we have

$$
\begin{aligned}
C_{d-3} \cdot C & =T_{P}^{d-1-l} \cdot C+L \cdot C+C_{l-3} \cdot C \\
& \geqq(d-1-l) d P+D_{r}>((d-2-l) d+l) P+D_{r} .
\end{aligned}
$$

(ii) By Lemma 2.3 we get $C_{d-3}=T_{P}^{d-m-1} C_{m-2}$. Hence, we have

$$
(d-m-1) d P+C_{m-2} \cdot C=(d-m-1) T_{P} \cdot C+C_{m-2} \cdot C \geqq(d-m-1) d P+D_{r},
$$

which implies that $C_{m-2} . C \geqq D_{r}$. Since $L . C \geqq D_{r}$, we have $L . C_{m-2} \geqq D_{r}$, which implies that $C_{m-2}=L C_{m-3}$. Thus, we obtain $C_{d-3}=T_{P}^{d-m-1} L C_{m-3}$. Hence, we have

$$
C_{d-3} \cdot C=(d-m-1) d P+L . C+C_{m-3} \cdot C \geqq(d-m-1) d P+P+D_{r}=t_{1} P+D_{r} .
$$

(iii) By Lemma 2.3 we obtain $C_{d-3}=T_{P}^{d-2-m} C_{m-1}$, which implies that $C_{m-1} . C \geqq P+D_{r}$. On the other hand, we have

$$
L_{1} \cdot C \geqq P+Q_{1}+\cdots+Q_{m-1} \quad \text { and } \quad L_{2} \cdot C \geqq P+Q_{m}+\cdots+Q_{2(m-1)},
$$

from which we get

$$
C_{m-1} \cdot L_{1} \geqq P+Q_{1}+\cdots+Q_{m-1} \quad \text { and } \quad C_{m-1} \cdot L_{2} \geqq P+Q_{m}+\cdots+Q_{2(m-1)} .
$$

Hence, we obtain $C_{m-1}=L_{1} L_{2} C_{m-3}$. Thus, we get

$$
\begin{aligned}
C_{d-3} \cdot C & =T_{P}^{d-2-m} L_{1} L_{2} C_{m-3} \cdot C \geqq T_{P}^{d-2-m} \cdot C+L_{1} \cdot C+L_{2} \cdot C \\
& \geqq((d-2-m) d+2) P+D_{r}=t_{1} P+D_{r} .
\end{aligned}
$$

(iv) Let $t_{i}=t_{2}$. By Lemma 2.3 we obtain $C_{d-3}=T_{P}^{d-m-1} C_{m-2}$, which implies that $C_{m-2} . C \geqq D_{r}$. Hence, we get $C_{m-2} \cdot L \geqq Q_{1}+\cdots+Q_{m-1}$, which implies that $C_{m-2}=L C_{m-3}$. Thus, we have $C_{d-3}=T_{P}^{d-m-1} L C_{m-3}$. Hence, we obtain

$$
C_{d-3} \cdot C=T_{P}^{d-m-1} \cdot C+L \cdot C+C_{m-3} \cdot C \geqq((d-m-1) d+1) P,
$$

which implies that $C_{d-3} \cdot C \geqq((d-m-1) d+1) P+D_{r}=t_{2} P+D_{r}$.

We have

$$
h^{0}\left(K-\left(t_{1}-1\right) P-Q_{1}\right)=h^{0}\left((d-1) P-Q_{1}\right)=1 .
$$


Hence, there is a unique effective divisor $E$ which is linearly equivalent to $(d-1) P-Q_{1}$. Then $E$ should be $Q_{2}+\cdots+Q_{m-1}+R_{m}+\cdots+R_{d-1}$, because we have

$$
d P=T_{P} . C \sim L . C=P+Q_{1}+Q_{2}+\cdots+Q_{m-1}+R_{m}+\cdots+R_{d-1} .
$$

Since $Q_{m}$ is different from $Q_{1}, \ldots, Q_{m-1}, R_{m}, \ldots, R_{d-1}$, we get

$$
h^{0}\left((d-1) P-Q_{1}-Q_{m}-Q_{2}-\cdots-Q_{m-1}\right)=0 .
$$

Thus, it follows that $0=h^{0}\left(K-\left(t_{1}-1\right) P-D_{r}\right)=h^{0}\left(K-t_{1} P-D_{r}\right)$.

(v) Let $t_{i}=t_{1}$. By Lemma 2.3 we obtain $C_{d-3}=T_{P}^{d-2-l} C_{l-1}$. Hence, we get $C_{l-1} . C \geqq(l-1) P+D_{r} . \quad$ Moreover, we have $L . C=Q_{1}+\cdots+Q_{r-1}+R_{l+1}$ $+\cdot+R_{d}$. Thus, we obtain $C_{l-1} . L \geqq Q_{1}+\cdots+Q_{r-1}$, which implies that $C_{l-1}=$ $L C_{l-2}$. Hence, we get $C_{d-3}=T_{P}^{d-2-l} L C_{l-2}$. Moreover, we have

$$
Q_{1}+\cdots+Q_{r-1}+R_{l+1}+\cdot+R_{d}+C_{l-2} \cdot C=L . C+C_{l-2} \cdot C \geqq(l-1) P+D_{r},
$$

which implies that $C_{l-2} . C \geqq(l-1) P+Q_{r}$. Hence, we get $C_{l-2} \cdot T_{P} \geqq(l-1) P$. Thus, we have $C_{l-2}=T_{P} C_{l-3}$. Hence, we get $C_{d-3}=T_{P}^{d-2-l} L T_{P} C_{l-3}$. Therefore, we obtain

$$
C_{d-3} \cdot C \geqq(d-2-l) d P+Q_{1}+\cdots+Q_{l}+d P+Q_{l+1}>t_{1} P+D_{r} .
$$

We have $K-\left(t_{2}-1\right) P \sim\left(d^{2}-3 d-d^{2}+4 d\right) P=d P$ where $t_{2}=(d-4) d+1$. Since $C$ is $(d-1)$-gonal, we get $h^{0}\left(d P-Q_{1}-Q_{2}\right)=1$. We note that $Q_{l+1}$ is general. Hence, we get $h^{0}\left(d P-Q_{1}-Q_{2}-Q_{l+1}\right)=0$. Thus, we get

$$
0=h^{0}\left(K-\left(t_{2}-1\right) P-D_{r}\right)=h^{0}\left(K-t_{2} P-D_{r}\right) .
$$

(vi) Let $t_{i}=t_{1}$. By Lemma 2.3 we have $C_{d-3}=T_{P}^{d-5} C_{2}$. Hence, we get C. $C_{2} \geqq Q_{1}+\cdots+Q_{5}$, which implies that $C_{2} . L \geqq Q_{1}+Q_{2}+Q_{3}$. Therefore, we obtain $C_{2}=L L_{Q_{4}, Q_{5}}$. Hence, we have

$$
\begin{aligned}
C_{d-3} \cdot C & =T_{P}^{d-5} \cdot C+L \cdot C+L_{Q_{4}, Q_{5}} \cdot C \\
& =d(d-5) P+P+Q_{1}+Q_{2}+Q_{3}+R_{4}+\cdots+R_{d-1}+L_{Q_{4}, Q_{5}} \cdot C,
\end{aligned}
$$

which implies that $C_{d-3} \cdot C \geqq t_{1} P+D_{r}$.

Suppose that there exists a curve $C_{d-3}$ such that $C_{d-3} \cdot C \geqq\left(t_{2}-1\right) P+D_{r}$, where $t_{2}=(d-5) d+3$. By Lemma 2.3 and the above method we have $C_{d-3}=$ $T_{P}^{d-5} L L_{Q_{4}, Q_{5}}$. Then we obtain

$$
(d-5) d+2 \leqq \operatorname{ord}_{P} C_{d-3} \cdot C=(d-5) d+1,
$$

which is a contradiction. Hence, we get

$$
0=h^{0}\left(K-\left(t_{2}-1\right) P-D_{r}\right)=h^{0}\left(K-t_{2} P-D_{r}\right) .
$$

(vii) We have

$$
h^{0}\left(K-\left(t_{1}-1\right) P-D_{r}\right)=h^{0}\left((2 d-1) P-\left(Q_{1}+\cdots+Q_{5}\right)\right)=5-5=0,
$$


because $Q_{1}, \ldots, Q_{5}$ are general points. It is enough to show that $h^{0}\left(K-\left(t_{2}-1\right) P-D_{r}\right)=0$, which is clear since $t_{2}=t_{1}+1$.

(viii) Let $t_{i}=t_{1}$. We get $C_{d-3}=T_{P}^{d-5} C_{2}$. Hence, we have $C . C_{2} \geqq Q_{1}$ $+\cdots+Q_{5}$. Since C. $L_{2} \geqq Q_{3}+Q_{4}+Q_{5}$, we have $C_{2}=L_{1} L_{2}$, which implies that

$$
1=h^{0}\left(K-\left(t_{1}-1\right) P-D_{r}\right)=h^{0}\left(K-t_{1} P-D_{r}\right)=h^{0}\left(K-t_{2} P-D_{r}\right) .
$$

SteP 5. By Step 2 the divisor $n P-2 Q_{1}-\cdots-2 Q_{r}$ is very ample. It follows from Step 4 and Theorem 2.2 in [9] that $H$ is DCP.

In the rest of this section we denote by $H$ a numerical semigoroup with $d_{2}(H)=$ $\langle 4,5\rangle$. Using Theorem 2.5 we will prove that the numerical semigroup $H$ is DCP. Let $n$ be the least odd number in $H$. In the following figure a cross $\times$ is one of the candidates of the elements $\mathbf{N}_{0} \backslash H$ which are odd numbers larger than $n$.

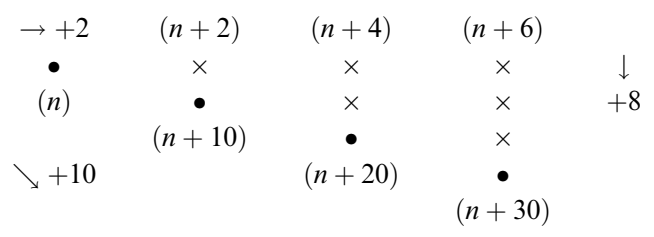

The candidates of odd gaps $>n$

In fact, for any odd $n \geqq 13$ we have

$$
S\left(2\langle 4,5\rangle+n \mathbf{N}_{0}\right)=\{8,10,20,30, n, n+10, n+20, n+30\}
$$

(see [7] if $n \geqq 15$ ). We note that $H \supseteqq 2\langle 4,5\rangle+n \mathbf{N}_{0}$.

Lemma 2.6. A numerical semigroup $H$ with $d_{2}(H)=\langle 4,5\rangle$ and the least odd number $n \geqq 13$ in $H$ is one of the following:

(a) $H_{n}=2\langle 4,5\rangle+n \mathbf{N}_{0}$, (b) $H_{n}+\langle n+2 t\rangle, t=1,2,3,6,7,11$,

(c) $H_{n}+\langle n+2 t, n+14\rangle, t=1,6$, (d) $H_{n}+\langle n+6, n+12\rangle$, (e) $H_{n}+\langle n+4$, $n+6\rangle$

(f) $H_{n}+\langle n+2, n+4, n+6\rangle$, (g) $H_{n}+\langle n+2, n+6\rangle$, (h) $H_{n}+\langle n+2, n+4\rangle$.

Proof. By the figure "The candidates of odd gaps $>n$ " we get the classification.

Applying Theorem 2.5 to the cases of Lemma 2.6 we get the following:

COROLlary 2.7. Let $H$ be a numerical semigroup of genus $\geqq 18$ with $d_{2}(H)=\langle 4,5\rangle$. Then $H$ is $D C P$.

Proof. We use the classification in Lemma 2.6, because the least odd number $n \leqq 11$ in $H$ implies $g(H) \leqq g\left(2\langle 4,5\rangle+n \mathbf{N}_{0}\right) \leqq 2 * 6+(11-1) / 2=17$. 
In the case (a) by Proposition 2.3 in [7] we get the result if $g(H) \geqq 18$.

In the case (b) we can apply Theorem 2.5 (i) to the cases $t=3,7,11$ if $g(H) \geqq 18$. We can apply Theorem 2.5 (ii) to the cases $t=1,6$ if $g(H) \geqq 18$. Theorem 2.5 (iii) is applied to the case $t=2$ if $g(H) \geqq 18$. We can apply Theorem 2.5 (iv), (v), (vii), (vi) and (viii) to the cases (c), (d), (e), (g) and (h) respectively if $g(H) \geqq 18$.

By Lemma 2.3 and Proposition 2.4 in [9] we get the result (f) if $g(H) \geqq 18$.

\section{Non-DCP numerical semigroups}

By [9] we know that any numerical semigroup $H$ of genus $g \geqq 9$ with $d_{2}(H)=\langle 3,5,7\rangle$ is DCP. We note that a point $P$ on a smooth plane curve $C$ of degree 4 with $H(P)=\langle 3,5,7\rangle$ satisfies $\operatorname{ord}_{P}\left(C \cdot T_{P}\right)=3$. But the following theorem shows that for any $d \geqq 5$ there is a numerical semigroup $H$ whose $d_{2}(H)$ is the Weierstrass semigroup of a point $P$ on a plane curve $C$ of degree $d$ with $\operatorname{ord}_{P} C \cdot T_{P}=d-1$ such that $H$ is not DCP. In this case we have

$d_{2}(H)=\langle d-1, d-1+d-2,2(d-1)+d-3, \ldots,(d-2)(d-1)+1\rangle($ see $[2])$, which is denoted by $H_{d}^{\prime}$. In this section we assume that $d \geqq 5$.

THEOREM 3.1. Let $n$ be an odd number with $n \geqq(d-2)(d-1)+1$. Assume that $H$ is $2 H_{d}^{\prime}+\langle n, n+2 t\rangle$ with $t=(d-3)(d-1)+2-l(d-1)$ for $l=1$ or 2. Then the semigroup $H$ is not DCP.

Proof. We have the standard basis $S\left(H_{d}^{\prime}\right)=\left\{d-1, s_{1}, \ldots, s_{d-2}\right\}$ for $H_{d}^{\prime}$, where $s_{i}=(d-1-i)(d-1)+i$ for all $i$. It follows from the condition $n \geqq$ $(d-2)(d-1)+1$ and Remark 2.1 in [7] that

$$
S\left(2 H_{d}^{\prime}+n \mathbf{N}_{0}\right)=\left\{2(d-1), 2 s_{1}, \ldots, 2 s_{d-2}\right\} \cup\left\{n, n+2 s_{1}, \ldots, n+2 s_{d-2}\right\} .
$$

By Remark 2.1 in [7] we have $g\left(2 H_{d}^{\prime}+n \mathbf{N}_{0}\right)=2 g\left(H_{d}^{\prime}\right)+(n-1) / 2$.

SteP 1. We obtain $g(H)=2 g\left(H_{d}^{\prime}\right)+(n-1) / 2-r$, where $r=1$ and 3 for $l=1$ and $l=2$ respectively. Indeed, if $l=1$, then the set $H \backslash\left(2 H_{d}^{\prime}+\langle n\rangle\right)$ consists of one element $n+2((d-3)(d-1)+2-(d-1))$, which implies that $r=1$. The semigroup $H$ with $l=2$ contains the following three elements $\circ$ in the figure below:

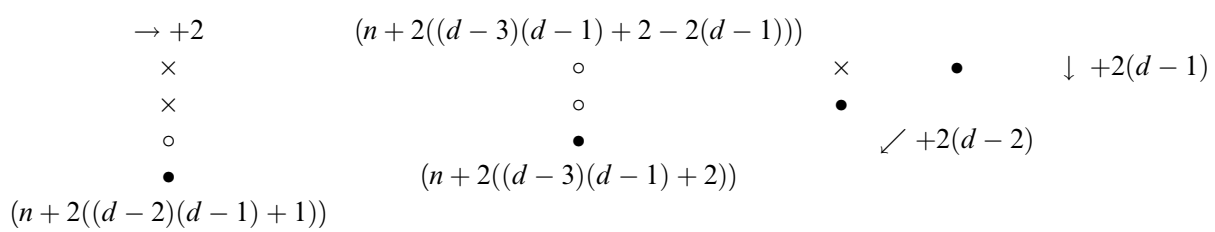

The elements of $H=2 H_{d}^{\prime}+n \mathbf{N}_{0}+(n+2((d-3)(d-1)+2-2(d-1))) \mathbf{N}_{0}$ 
Assume that there is a double covering $\pi: \tilde{C} \rightarrow C$ with a ramification point $\tilde{P}$ over a point $P$ with $H(\tilde{P})=H$.

SteP 2. There are $r$ points $Q_{1}, \ldots, Q_{r}$ distinct from $P$ such that $2 D$ is linearly equivalent to a reduced divisor containing $P$, where $D=\frac{n+1}{2} P-D_{r}$ with $D_{r}=Q_{1}+\cdots+Q_{r}$.

STEP 3. We show that the equality $h^{0}\left(K-t P-D_{r}\right)=h^{0}\left(K-(t-1) P-D_{r}\right)$ induces a contradiction. Let $T_{P} . C=(d-1) P+Q$ with $Q \neq P$.

First, let $l=1$. We consider the case $Q_{1}=Q$. Let $C_{d-3}=T_{P}^{d-4} L$ with a line $L$ passing through $P$ with $L \neq T_{P}$. Then in view of $d \geqq 5$ we have

$$
\begin{aligned}
C_{d-3} . C & =(d-4)(d-1) P+(d-4) Q_{1}+L . C \\
& \geqq(d-4)(d-1) P+Q_{1}+P=((d-4)(d-1)+1) P+Q_{1}
\end{aligned}
$$

and $C_{d-3} . C \nsupseteq((d-4)(d-1)+2) P$. This is a contradiction.

We consider the case with $Q_{1} \neq Q$. We set $C_{d-3}=T_{P}^{d-4} L$ with the line $L$ passing through $P$ and $Q_{1}$ which is a reducible curve of degree $d-3$. In view of $Q_{1} \neq Q$ we note that $L \neq T_{P}$. Then

$$
\begin{aligned}
C_{d-3} \cdot C & =T_{P}^{d-4} \cdot C+L . C=(d-4)(d-1) P+(d-4) Q+L . C \\
& \geqq(d-4)(d-1) P+(d-4) Q+P+Q_{1} \geqq((d-4)(d-1)+1) P+Q_{1} .
\end{aligned}
$$

But $C_{d-3} . C \nsupseteq((d-4)(d-1)+2) P$. This is a contradiction.

Next, let $l=2$. We consider the case $Q_{1}=Q_{2}=Q_{3}=Q$.

Let $d=5$. Assume that $h^{0}(K-P-3 Q)=h^{0}(K-2 P-3 Q)$. Let $C_{2}$ be a conic such that $C_{2} . C \geqq 2 P+3 Q$. Then $C_{2} \cdot T_{P} \geqq 2 P+Q$. Hence, we get $C_{2}=T_{P} L$ where $L$ is a line. Moreover, we have

$$
2 P+3 Q \leqq C_{2} . C=T_{P} L . C=T_{P} \cdot C+L . C=4 P+Q+L . C,
$$

which implies that $L=T_{Q}$. Hence, we get $C_{2}=T_{P} T_{Q}$. Thus, we obtain

$$
1=h^{0}(K-2 P-3 Q)=h^{0}(K-P-3 Q)=6+1-6+h^{0}(P+3 Q) \geqq 2,
$$

which is a contradiction.

Let $d \geqq 6$. Let $L_{1}$ be a line through $P$ which is distinct from $T_{P}$. We set $L_{0}=T_{Q}$. Then in view of $d \geqq 6$ we have

$$
\begin{aligned}
T_{P}^{d-5} L_{1} L_{0} \cdot C & \geqq(d-5)(d-1) P+(d-5) Q+P+2 Q \\
& =((d-5)(d-1)+1) P+(d-3) Q \geqq((d-5)(d-1)+1) P+3 Q .
\end{aligned}
$$

But we get $T_{P}^{d-5} L_{1} L_{0} . C ¥((d-5)(d-1)+2) P$. This is a contradiction.

We consider the case $Q_{1} \neq Q$ when we renumber $Q_{1}, Q_{2}$ and $Q_{3}$. Let $L_{0}$ be the line such that $L_{0} . C \geqq Q_{2}+Q_{3}$. If $L_{0} \ni P$, then we take $L_{1}$ as a line through 
$Q_{1}$ and not containing $P$. If $L_{0} \not P$, then we take $L_{1}$ as the line through $Q_{1}$ and $P$. Then we get

$$
L_{0} L_{1} T_{p}^{d-5} \cdot C \geqq((d-5)(d-1)+1) P+Q_{1}+Q_{2}+Q_{3}
$$

and $L_{0} L_{1} T_{p}^{d-5} . C \nsupseteq((d-5)(d-1)+2) P$. This is a contradiction.

In the case $d=5$ we get the following by Theorem 3.1:

COROllary 3.2. Set $H(n)=2\langle 4,7,10,13\rangle+n \mathbf{N}_{0}$, where $n$ is an odd number with $n \geqq 13$. Then neither $H(n)+\langle n+4\rangle$ nor $H(n)+\langle n+12\rangle$ is $D C P$.

\section{Double coverings of plane curves of degree 5}

In this section $H$ denotes a numerical semigroup with $d_{2}(H)=\langle 4,7,10,13\rangle$. Let $n$ be the least odd number in $H$. Then we note that $g(H) \leqq 12+(n-1) / 2$ (for example, see Lemma 3.1 in [4]). Assume that $n \geqq 13$. In the following figure a cross $\times$ is one of the candidates of the odd numbers in $\mathbf{N}_{0} \backslash H$ which are larger than $n$.

$\begin{array}{ccccc}\rightarrow+2 & (n+2) & (n+4) & (n+6) & \\ \bullet & \times & \times & \times & \downarrow \\ (n) & \times & \times & \bullet & +8 \\ & \times & \bullet & (n+14) & \\ & \bullet & (n+20) & \swarrow+6 & \\ & (n+26) & & & \end{array}$

The candidates of odd gaps $>n$

We get $6+(n-1) / 2 \leqq g(H) \leqq 12+(n-1) / 2$ by Lemma 2.2 in [7]. Hence, we set $g(H)=12+(n-1) / 2-r$ with $0 \leqq r \leqq 6$. By the above figure "The candidates of odd gaps $>n$ " the numerical semigroups $H$ are determined as follows: lowing:

Lemma 4.1. Set $H(n)=2\langle 4,7,10,13\rangle+n \mathbf{N}_{0}$. Then $H$ is one of the fol-

(i) If $g(H)=12+(n-1) / 2$, then $H=H(n)$.

(ii) If $g(H)=11+(n-1) / 2$, then $H$ is either

1) $H(n)+\langle n+6\rangle$ or 2) $H(n)+\langle n+12\rangle$ or 3$) H(n)+\langle n+18\rangle$.

(iii) If $g(H)=10+(n-1) / 2$, then $H$ is either

1) $H(n)+\langle n+6, n+12\rangle$ or 2$) H(n)+\langle n+6, n+18\rangle$ or 3$) H(n)+\langle n+10\rangle$ or 4) $H(n)+\langle n+12, n+18\rangle$.

(iv) If $g(H)=9+(n-1) / 2$, then $H$ is either

1) $H(n)+\langle n+2\rangle$ or 2) $H(n)+\langle n+4\rangle$ or 3) $H(n)+\langle n+6, n+10\rangle$ or 4) $H(n)+\langle n+6, n+12, n+18\rangle$ or 5$) H(n)+\langle n+10, n+12\rangle$.

(v) If $g(H)=8+(n-1) / 2$, then $H$ is either

1) $H(n)+\langle n+2, n+6\rangle$ or 2) $H(n)+\langle n+2, n+12\rangle$ or 3$) H(n)+\langle n+4$, $n+6\rangle$ or 4$) H(n)+\langle n+4, n+10\rangle$ or 5$) H(n)+\langle n+6, n+10, n+12\rangle$. 
(vi) If $g(H)=7+(n-1) / 2$, then $H$ is either

1) $H(n)+\langle n+2, n+4\rangle$ or 2) $H(n)+\langle n+2, n+6, n+12\rangle$ or 3$) H(n)+$ $\langle n+4, n+6, n+10\rangle$.

(vii) If $g(H)=6+(n-1) / 2$, then $H=H(n)+\langle n+2, n+4, n+6\rangle$.

THEOREM 4.2. If $g=g(H) \geqq 18$, then the numerical semigroup $H$ except for (ii) 2) and (iv) 2) is $D C P$.

Proof. We give the proofs according to the cases given in Lemma 4.1. Let $(C, P)$ be a pointed plane curve with $H(P)=\langle 4,7,10,13\rangle$. Then we have $T_{P}(C) . C=4 P+R$ with some point $R \neq P$, which implies that $K \sim 8 P+2 R$. To show that $H$ is DCP we use Theorem 2.2 in [9]. So, we need to choose $r$ points $Q_{1}, \ldots, Q_{r}$ of $C$ satisfying the assumptions of the theorem in [9]. We set $D=\frac{n+1}{2} P-Q_{1}-\cdots-Q_{r}$. Then we note that

$$
\operatorname{deg}(2 D-P)=n-2 r=2 g-23 \geqq 36-23=13
$$

because $g(H) \geqq 18$. Hence, the divisor $2 D-P$ is very ample.

In the case (i) it follows from Proposition 2.3 in [7] that $H$ is DCP.

We consider the case (ii) 1). Let $Q_{1}=R$. Since $C$ is not trigonal, we get $h^{0}(2 P+R)=1$. It is clear that $h^{0}(3 P+R)=2$ since $|4 P+R|$ is a net without base points. Thus, we get the result. Theorem 3.1 implies that $H$ is not DCP in the case (ii) 2). In the case (ii) 3) it follows from Proposition 2.4 in [7] that $H$ is DCP.

Let $H$ be the semigroup in the case (iii) 1). We set $Q_{1}=Q_{2}=R$. We have

$$
\begin{aligned}
& h^{0}(2 P+2 R)=4+1-6+h^{0}(6 P)=1 \quad \text { and } \\
& h^{0}(3 P+2 R)=5+1-6+h^{0}(5 P)=2 .
\end{aligned}
$$

Moreover, we get

$$
\begin{aligned}
& h^{0}(5 P+2 R)=7+1-6+h^{0}(3 P)=3 \text { and } \\
& h^{0}(6 P+2 R)=8+1-6+h^{0}(2 P)=4 .
\end{aligned}
$$

In the case (iii) 2) we take a general point $Q$. Let $Q_{1}=R$ and $Q_{2}=Q$. Then we have $h^{0}(9 P+R+Q)=6$ and $h^{0}(8 P+R+Q)=5$, because of $8 P+R+$ $Q \nsucc 8 P+2 R \sim K$. Moreover, we get $h^{0}(2 P+R+Q)=-1+h^{0}(6 P+R-Q)$. Now we have

$$
h^{0}(6 P+R)=2+h^{0}(2 P+R)=3,
$$

because $C$ is 4-gonal. Hence, we get $h^{0}(2 P+R+Q)=-1+2=1$, because $Q$ is general. We see that $h^{0}(3 P+Q+R)=2$ since $|4 P+R|$ is a net and $h^{0}(2 P+R+Q)=1$.

In the case (iii) 3 ) we have

$$
h^{0}(K-5 P)=h^{0}(5 P)=2 \text { and } h^{0}(K-6 P)=-1+h^{0}(6 P)=1 .
$$


Let $Q_{1}$ be a general point. Since $h^{0}\left(K-5 P-Q_{1}\right)=1$, there exists a unique effective divisor $E=S_{1}+S_{2}+S_{3}+S_{4}$ of degree 4 with $E \sim K-5 P-Q_{1}$. The effective divisor $E$ does not contain $P$, because $h^{0}(K-6 P)=1$. Moreover, we have $E \neq 4 R$. Indeed, assume that $E=4 R$. Then we get

$$
4 R \sim K-5 P-Q_{1} \sim 3 P+2 R-Q_{1},
$$

which implies that $2 R+Q_{1} \sim 3 P$. This contradicts $h^{0}(3 P)=1$. We may assume that $S_{4} \neq R$ and $S_{4} \neq P$. We set $Q_{2}=S_{4}$. Then we have

$$
h^{0}\left(K-5 P-Q_{1}-Q_{2}\right)=h^{0}\left(S_{1}+S_{2}+S_{3}\right)=1 \text {. }
$$

Hence, there exists a unique conic $C_{2}$ with $C . C_{2} \geqq 5 P+Q_{1}+Q_{2}$. Take a conic $C_{2}^{\prime}$ with $C . C_{2}^{\prime} \geqq 4 P+Q_{1}+Q_{2}$. Since $Q_{1}$ and $Q_{2}$ are different from $R$, we must have $C_{2}^{\prime}=T_{P} L_{Q_{1}, Q_{2}}$, where $L_{Q_{1}, Q_{2}}$ is the line through $Q_{1}$ and $Q_{2}$. Hence, we obtain $h^{0}\left(K-4 P-Q_{1}-Q_{2}\right)=1$.

In the case (iii) 4) let $Q_{1}$ and $Q_{2}$ be general points. Then we have

$$
h^{0}\left(9 P+Q_{1}+Q_{2}\right)=6 \text { and } h^{0}\left(8 P+Q_{1}+Q_{2}\right)=5,
$$

because $8 P+Q_{1}+Q_{2} \nsucc K$. Since $h^{0}(3 P+2 R)=h^{0}(5 P)=2$, we obtain

$$
\begin{aligned}
& h^{0}\left(5 P+Q_{1}+Q_{2}\right)=2+h^{0}\left(3 P+2 R-Q_{1}-Q_{2}\right)=2 \text { and } \\
& h^{0}\left(6 P+Q_{1}+Q_{2}\right)=3 .
\end{aligned}
$$

Let $H$ be the semigroup in the case (iv) 1). We take a line $L_{P}$ through $P$ distinct from $T_{P}$. Then we have $L_{P} . C=P+S_{1}+S_{2}+S_{3}+S_{4}$. We set $Q_{i}=S_{i}$ for all $i=1,2,3$. It is clear that $h^{0}(4 P+R)=3$ and $h^{0}\left(P+Q_{1}+Q_{2}+Q_{3}\right)=2$ by the choice of $R$ and $Q_{i}$ 's.

In the case (iv) 2) $H$ is not DCP by Theorem 3.1 .

We consider the case (iv) 3). Let $L_{P}$ be a line as in the case (iv) 1 ). We set $Q_{1}=R, Q_{2}=S_{3}$ and $Q_{3}=S_{4}$. Then we have

$$
\begin{aligned}
h^{0}(K & \left.-5 P-Q_{1}-Q_{2}-Q_{3}\right) \\
& =h^{0}\left(4 P+R+P+S_{1}+S_{2}+Q_{2}+Q_{3}-5 P-Q_{1}-Q_{2}-Q_{3}\right) \\
& =h^{0}\left(S_{1}+S_{2}\right)=1 .
\end{aligned}
$$

Moreover, it is enough to show that $h^{0}\left(K-2 P-Q_{1}-Q_{2}-Q_{3}\right)=1$, which is clear by the choice of $Q_{i}$ 's.

Let $H$ be the semigroup in the case (iv) 4). We set $Q_{1}=R$. Take two general points $Q_{2}$ and $Q_{3}$. We have

$$
h^{0}\left(9 P+Q_{1}+Q_{2}+Q_{3}\right)=7=h^{0}\left(8 P+Q_{1}+Q_{2}+Q_{3}\right)+1 .
$$

Moreover, we have

$$
h^{0}\left(6 P+Q_{1}+Q_{2}+Q_{3}\right)=4+h^{0}\left(2 P+R-Q_{2}-Q_{3}\right)=4
$$

and

$$
h^{0}\left(5 P+Q_{1}+Q_{2}+Q_{3}\right)=3+h^{0}\left(3 P+R-Q_{2}-Q_{3}\right)=3,
$$


because $Q_{2}$ and $Q_{3}$ are general. Let $C_{2}$ be a conic with $C_{2} . C \geqq 2 P+Q_{1}+$ $Q_{2}+Q_{3}$. Then $C_{2}$ is uniquely determined. Hence, we get

$$
h^{0}\left(2 P+Q_{1}+Q_{2}+Q_{3}\right)=1 \text { and } h^{0}\left(3 P+Q_{1}+Q_{2}+Q_{3}\right)=2 .
$$
have

We are in the case (iv) 5). Let $Q_{1}, Q_{2}$ and $Q_{3}$ be general points of $C$. We

$$
h^{0}\left(6 P+Q_{1}+Q_{2}+Q_{3}\right)=4+h^{0}\left(2 P+2 R-Q_{1}-Q_{2}-Q_{3}\right)=4 .
$$

In view of $h^{0}(3 P+2 R)=h^{0}(5 P)=2$ we have $h^{0}\left(5 P+Q_{1}+Q_{2}+Q_{3}\right)=3$. Moreover, we get $h^{0}(4 P+2 R)=1+h^{0}(4 P)=3$, which implies that

$$
h^{0}\left(4 P+Q_{1}+Q_{2}+Q_{3}\right)=2 .
$$

We consider the case (v). We note that by Namba's Theorem we have $h^{0}\left(Q_{1}+Q_{2}+Q_{3}+Q_{4}\right)=1$ if four points $Q_{1}, Q_{2}, Q_{3}$ and $Q_{4}$ of $C$ do not lie on a line.

In the case (v) 1) let $L_{P}$ be a line through $P$ with $L_{P} \neq T_{P}$. We set $L_{P} . C=$ $P+Q_{1}+Q_{2}+Q_{3}+S$ and $Q_{4}=R$. Let $C_{2}$ be a conic with $C_{2} . C \geqq P+Q_{1}+$ $Q_{2}+Q_{3}+Q_{4}$. Then we get $C_{2} \cdot L_{P} \geqq P+Q_{1}+Q_{2}+Q_{3}$. Hence, we have $C_{2}=$ $L_{P} L$ where $L$ is any line through $Q_{4}$, which implies that $h^{0}\left(K-P-Q_{1}-\right.$ $\left.Q_{2}-Q_{3}-Q_{4}\right)=2$. Let $C_{2}^{\prime}$ be a conic with $C_{2}^{\prime} . C \geqq 2 P+Q_{1}+Q_{2}+Q_{3}+Q_{4}$. Then we have $C_{2}^{\prime} \cdot L_{P} \geqq Q_{1}+Q_{2}+Q_{3}+P$. In view of $Q_{4}=R$ we get $C_{2}^{\prime}=$ $L_{P} T_{P}$, which implies that $h^{0}\left(K-2 P-Q_{1}-Q_{2}-Q_{3}-Q_{4}\right)=1$. It is clear that $h^{0}\left(K-3 P-Q_{1}-Q_{2}-Q_{3}-Q_{4}\right)=1 \quad$ since $C_{2}^{\prime} . C=L_{P} T_{P} . C \geqq 5 P+C_{1}+C_{2}+$ $C_{3}+C_{4}$.

We are in the case (v) 2). Let $L_{P}$ be a line through $P$ with $L_{P} \neq T_{P}$. We set $L_{P} . C=P+Q_{1}+Q_{2}+Q_{3}+S$. Let $Q_{4}$ be a point of $C$ not on the line $L_{P}$ with $Q_{4} \neq R$. Then we have

$$
\begin{aligned}
h^{0}( & P \\
& \left.+Q_{1}+Q_{2}+Q_{3}+Q_{4}\right) \\
& =4+h^{0}\left(K-5 P-Q_{1}-Q_{2}-Q_{3}-Q_{4}\right) \\
& =4+h^{0}\left(5 P+R+Q_{1}+Q_{2}+Q_{3}+S-5 P-Q_{1}-Q_{2}-Q_{3}-Q_{4}\right) \\
& =4+h^{0}\left(R+S-Q_{4}\right)=4 .
\end{aligned}
$$

Moreover, we get $h^{0}\left(6 P+Q_{1}+Q_{2}+Q_{3}+Q_{4}\right)=5$. It is clear that $h^{0}\left(P+Q_{1}+\right.$ $\left.Q_{2}+Q_{3}+Q_{4}\right)=2$ since the four points $P, Q_{1}, Q_{2}, Q_{3}$ lie on the line $L_{P}$ and $Q_{4} \notin L_{P}$.

Let $H$ be the semigroup in the case (v) 3). We take a line $L$ containing neither $P$ nor $R$. We set $L . C=Q_{1}+Q_{2}+Q_{3}+S+T$ and $Q_{4}=R$. Let $C_{2}$ be a conic with $C_{2} \cdot C \geqq P+Q_{1}+Q_{2}+Q_{3}+Q_{4}$. Then $C_{2} \cdot L \geqq Q_{1}+Q_{2}+Q_{3}$. Hence, we get $C_{2}=L T_{P}$. We note that $C . C_{2} \geqq 4 P+Q_{1}+Q_{2}+Q_{3}+Q_{4}$.

We consider the case (v) 4). Let $L_{1}$ be a line through $P$ with $L_{1} \neq T_{P}$ such that $L_{1} . C=P+Q_{1}+Q_{2}+S_{1}+T_{1}$. Let $L_{2}$ be a line through $P$ different from 
$T_{P}$ and $L_{1}$ such that $L_{2} . C=P+Q_{3}+Q_{4}+S_{2}+T_{2}$. Then $h^{0}\left(K-4 P-Q_{1}-\right.$ $\left.Q_{2}-Q_{3}-Q_{4}\right)=0$ since $L_{1} L_{2}$ is the only conic passing through $P$ and all $Q_{i}$ 's. Hence, we get

$$
h^{0}\left(5 P+Q_{1}+Q_{2}+Q_{3}+Q_{4}\right)=4 \text { and } h^{0}\left(4 P+Q_{1}+Q_{2}+Q_{3}+Q_{4}\right)=3 .
$$

On the other hand, let $C_{2}^{\prime}$ be a conic with $C_{2}^{\prime} . C \geqq P+Q_{1}+Q_{2}+Q_{3}+Q_{4}$. Then we have $C_{2}^{\prime} \cdot L_{1} \geqq P+Q_{1}+Q_{2}$. Hence, we obtain $C_{2}^{\prime}=L_{1} L^{\prime}$ where $L^{\prime}$ is the line with $L^{\prime} . C \geqq Q_{3}+Q_{4}$. The line $L^{\prime}$ must be $L_{2}$. Thus, $C_{2}^{\prime}$ is uniquely determined. Moreover, we get $C_{2}^{\prime} . C \geqq 2 P+Q_{1}+Q_{2}+Q_{3}+Q_{4}$.

Let $H$ be the semigroup in the case (v) 5). We set $Q_{1}=Q_{2}=R$. Let $Q_{3}$ and $Q_{4}$ be general points of $C$. Then we have

$$
h^{0}\left(K-2 P-Q_{1}-Q_{2}-Q_{3}-Q_{4}\right)=h^{0}\left(6 P-Q_{3}-Q_{4}\right)=0 .
$$

We consider the case (vi) 1). Let $Q_{1}, Q_{2}$ and $Q_{3}$ be general points of $C$. Then we have $h^{0}\left(K-2 P-Q_{1}-Q_{2}-Q_{3}\right)=1$. Hence there is a unique conic $C_{2}$ with $C_{2} . C \geqq 2 P+Q_{1}+Q_{2}+Q_{3}$, which is irreducible, because $T_{P}$ does not contain any $Q_{i}$ and no three of the four points $P, Q_{1}, Q_{2}$ and $Q_{3}$ are collinear. Let $C_{2} . C=2 P+Q_{1}+Q_{2}+Q_{3}+Q_{4}+Q_{5}+S_{1}+S_{2}+S_{3}$. Here, we have $Q_{i} \neq P$ for all $i$ and $S_{j} \neq P$ for all $j$, because $C_{2}$ is irreducible. Then we get

$$
h^{0}\left(K-2 P-Q_{1}-Q_{2}-Q_{3}-Q_{4}-Q_{5}\right)=1 .
$$

Moreover, let $C_{2}^{\prime}$ be a conic with $C_{2}^{\prime} . C \geqq Q_{1}+Q_{2}+Q_{3}+Q_{4}+Q_{5}$. Then $C_{2} . C_{2}^{\prime} \geqq Q_{1}+Q_{2}+Q_{3}+Q_{4}+Q_{5}$. Since $C_{2}$ is irreducible, we must have $C_{2}^{\prime}=C_{2}$. Hence, we get $1=h^{0}\left(K-Q_{1}-Q_{2}-Q_{3}-Q_{4}-Q_{5}\right)$.

Let $H$ be the semigroup in the case (vi) 2). We take general points $Q_{1}, Q_{2}$, $Q_{3}$ and $Q_{4}$ of $C$. We have $h^{0}\left(K-2 P-Q_{1}-Q_{2}-Q_{3}-Q_{4}\right)=0$, because $Q_{1}$, $Q_{2}, Q_{3}$ and $Q_{4}$ are general. Since $h^{0}\left(K-P-Q_{1}-Q_{2}-Q_{3}-Q_{4}\right)=1$, there is a unique effective divisor $E$ which is linearly equivalent to $K-P-Q_{1}-Q_{2}-$ $Q_{3}-Q_{4}$. We have $E \neq 5 P$, because $h^{0}(2 P+2 R)=1$. We take a point $Q_{5}$ with $Q_{5} \neq P$ such that $E \geqq Q_{5}$. Then we get

$$
h^{0}\left(K-P-Q_{1}-Q_{2}-Q_{3}-Q_{4}-Q_{5}\right)=h^{0}\left(E-Q_{5}\right)=1 .
$$

Since no four points of $Q_{1}, Q_{2}, Q_{3}, Q_{4}$ and $Q_{5}$ are collinear, there exists a unique conic passing through all $Q_{i}$ 's. Thus, we get $h^{0}\left(K-Q_{1}-Q_{2}-Q_{3}-\right.$ $\left.Q_{4}-Q_{5}\right)=1$. we have

In the case (vi) 3) let $Q_{1}, Q_{2}, Q_{3}, Q_{4}$ and $Q_{5}$ be general points of $C$. Then

$$
h^{0}\left(K-P-Q_{1}-Q_{2}-Q_{3}-Q_{4}-Q_{5}\right)=0 .
$$

In the case (vii) we get the result by Corollary 2.8 in [7].

Acknowledgement. The first author is partially supported by Basic Science Research Program through the National Research Foundation of Korea (NRF) funded by the Ministry of Education, Science and Technology 
(2012R1A1A2042228). The second author, who is the corresponding author, is partially supported by Grant-in-Aid for Scientific Research (24540057), Japan Society for the Promotion Science. We thank the referee for many helpful comments that improved the clarity of the paper.

\title{
REFERENCES
}

[ 1 ] M. Coppens AND T. Kato, The gonality of smooth curves with plane models, Manuscripta Mathematica 70 (1990), 5-25.

[2] M. Coppens AND T. KATO, The Weierstrass gap sequences at an inflection point on a nodal plane curve, aligned inflection points on plane curves, Bollettino U. M. I. 11-B (1997), 1-33.

[ 3 ] S. Greco And G. Raciti, The Lüroth semigroup of plane algebraic curves, Pacific J. Math. 151 (1991), 43-56.

[4] T. Harui and J. Komeda, Numerical semigroups of genus eight and double coverings of curves of genus three, Semigroup Forum 89 (2014), 571-581.

[5] T. Harui and J. Komeda, Numerical semigroups of genus seven and double coverings of curves of genus three, to appear in Semigroup Forum, doi:10.1007/s00233-014-9621-0.

[6] T. Harui and J. Komeda, Numerical semigroups of genus six and double coverings of curves of genus three, to appear in Semigroup Forum, doi:10.1007/s00233-014-3671-3.

[7] S. J. Kim AND J. KomedA, Weierstrass semigroups on double covers of genus four curves, J. Algebra 405 (2014), 142-167.

[8] J. Komeda, A numerical semigroup from which the semigroup gained by dividing by two is either $\mathbf{N}_{0}$ or a 2-semigroup or $\langle 3,4,5\rangle$, Research Reports of Kanagawa Institute of Technology B-33 (2009), 37-42.

[ 9 ] J. KomedA, On Weierstrass semigroups of double coverings of genus three curves, Semigroup Forum 83 (2011), 479-488.

[10] J. Komeda, Double coverings of curves and non-Weierstrass semigroups, Communications in Algebra 41 (2013), 312-324.

[11] J. Komeda And A. OHbuchi, On double coverings of a pointed non-singular curve with any Weierstrass semigroup, Tsukuba J. Math. 31 (2007), 205-215.

[12] M. NAmBA, Families of meromorphic functions on compact Riemann surfaces, Lecture notes in math. 767, Springer-Verlag, Berlin, 1979.

\author{
Seon Jeong Kim \\ Department of Mathematics AND RINS \\ GYEONGSANG NATIONAL UNIVERSITY \\ JINJU, 660-701 \\ REPUBLIC OF KOREA \\ E-mail: skim@gnu.ac.kr \\ Jiryo Komeda \\ Department of Mathematics \\ Center for Basic Education and Integrated Learning \\ Kanagawa Institute of Technology \\ Atsugi, Kanagawa, 243-0292 \\ JAPAN \\ E-mail: komeda@gen.kanagawa-it.ac.jp
}

